\title{
Integrating Metabolome and Functional Data in Plant Pathology and Protection Studies
}

\section{Conceição Santos*}

CESAM and Laboratory of Biotechnology and Cytometry, University Aveiro 3810-193 Aveiro, Portugal

\section{Editorial}

The dynamic interactions that are established between plant and microorganisms rely on complex mechanisms of recognition and defense. Primary metabolism has been traditionally implicated as a major target in several plant-pathogen interactions, but also critical changes in some pathways of secondary metabolism have been identified [1]. In the last decade, transcriptomics has largely contributed to decipher some of the cellular pathways that are involved in recognition and defense mechanisms between hosts-microorganisms interactions. For example, infection has been associated with differential regulation of genes involving energy processing (eg., up-regulation of genes related with energy production while down-regulation of genes associated with assimilatory processes as photosynthesis or chlorophyll biosynthesis). These and other transcriptomic findings contributed to reevaluate the role of some metabolic pathways during host-pathogen interactions, namely at modulating signal transduction cascades [2].

Currently, attention is being given to the valuable information that mebabolomics may deliver on plant responses to environmental challenges. Metabolomics is a multidisciplinary discipline gathering for example biology, chemistry, mathematics and informatics. Major techniques used on metabolite profiles include Gas Chromatography (GC), High Performance Liquid Chromatography (HPLC), Mass Spectrometry (MS), Nuclear Magnetic Resonance (NMR), Fourier Transform Infrared Spectroscopy (FT-IR), Molecular Spectroscopy (MS), and the generated data is treated with multivariate analysis. For a specific biological system, the huge amount of metabolic data then generated allows one to establish qualitative and quantitative profiling of metabolites, and simultaneously to establish a crucial link between that particular genotype and phenotype. However, in plant-environment interactions, metabolomic approaches require the optimization of reliable metabolomics/computational biology protocols, so warranting a robust analysis of that specific interaction. Also, the true value of metabolomics relies on its integration into a broader system biology approach, to better understand plant's performance and production. Despite its huge potential, the use of metabolomic tools in plant phytopathology and pest control studies is in its infancy. Next, I will highlight some pioneering metabolomics/functional approaches plant pathology and defense.

GC-MS analyses of soybean roots infected with Fusarium tucumaniae showed an increase of the levels of several amino acids, contributing to better understand the signaling networks involved the development of the sudden death syndrome in susceptible soybean plants [3]. Also, ultrahigh Performance Liquid Chromatography (UPLC) Linked Quadrupole Time-Of-Flight Mass Spectrometry (QTOFMS) were used to profile the metabolome of Cirsium arvense leaves infected with the endophyte Chaetomium cochlioides, and showed substantial biochemical changes (eg., oxilipins) and the identified new metabolites in this species; also metabolomics profile helped to understand that some chemical responses elicited by these infected plants were similar to those following wounding, or herbivory or pathogen invasion [4]. Also GC-MS and LC-MS showed that the profiles of isoflavone aglycones and glycoconjugate changed in lupin leaves when infected with the anthracnose fungus Colletotrichum lupini [5].

We can also find interesting examples of metabolomics tools applied to woody plants pathological studies. For example, HPLC-MS analyses demonstrated that Candidatus liberibacter asiaticus induced changes in a citrus crop metabolome profile, namely affecting flavonoid glycosides, polymethoxylated flavones, and hydroxycinnamates levels [6]. Also we have combined functional data with FT-IR and GC analyses to determine the profiles of some carbohydrates in in vitro elms challenged with Ophiostoma ulmi, and integrate those responses with physiological data [7]. On other hand, Vitis pathology represents an interesting case study of the increasing contributions of metabolomics to decipher plant responses to pathogens. The use of (1) H Nuclear Magnetic Resonance (NMR) spectroscopy showed differences in metabolic profiles between healthy and botrytized berries [8]. Similarly, Ali et al. [9] compared by MNR the metabolic profiles of sensitive $v s$. resistant grapevine cultivars in response to downy mildew infection, and found that major discriminatory classes were organic acids, amino acids, carbohydrates, phenylpropanoids and flavonoids. The authors also correlated quercetin-3-O-glucoside and a transferuloyl derivative with resistance. More recently, MNR contributed to identify some changes in metabolome that occur in grapevine plants with esca syndrome. These plants showed higher levels of phenolic compounds and decreases of carbohydrates [10] - which supported previous findings of decreased photosynthetic activities [11]-, and authors suggested that diseased plants are rerouting carbon and energy from primary to secondary metabolism [10].

Likewise, integration of metabolomics data in plant's physiological responses to pesticides is emerging. For example, using a High Resolution Magic Angle Spinning (HR-MAS) NMR metabolomic study of Lactuca sativa leaves, we have recently demonstrated that mancozeb induced changes in amino acids, fumarate and malate, suggesting Krebs cycle up-regulation [12].

Finally, multiple functional and genetic techniques have also offered (particularly when combined) excellent data to complement metabolomics. Combining all together will contribute to design more realistic cell functional models. Physiological combined perspectives,

*Corresponding author: Conceição santos, CESAM and Laboratory of Biotechnology and Cytometry, University Aveiro 3810-193 Aveiro, Portugal, Tel: (+351) 234370766; Ext: 22733; E-mail: csantos@ua.pt

Received December 03, 2014; Accepted December 06, 2014; Published December 09, 2014

Citation: Santos C (2015) Integrating Metabolome and Functional Data in Plant Pathology and Protection Studies. J Plant Pathol Microb 6: e108. doi:10.4172/2157$7471.1000 \mathrm{e} 108$

Copyright: (c) 2015 Santos C. This is an open-access article distributed under the terms of the Creative Commons Attribution License, which permits unrestricted use, distribution, and reproduction in any medium, provided the original author and source are credited. 
Citation: Santos C (2015) Integrating Metabolome and Functional Data in Plant Pathology and Protection Studies. J Plant Pathol Microb 6: e108. doi:10.4172/2157-7471.1000e108

Page 2 of 2

however, have up to moment been widely applied to plant-abiotic stress relations $[13,14]$ and have enormous potential for plant-pathogen interactions. If achieved, this global functional perspective will certainly contribute to other plant fields of research, including biotechnology, breeding and agrochemistry, with ultimate consequences in agro-food production and phytopharmaceutical industries.

\section{References}

1. Bednarek $P$ (2012) Chemical warfare or modulators of defence responses - the function of secondary metabolites in plant immunity.Curr Opin Plant Biol 15 407-14

2. Rojas, C, Senthil-Kumar M, Tzin V, Mysore K (2014) Regulation of primary plant metabolism during plant-pathogen interactions and its contribution to plant defense. Frontiers in Plant Science 5: 17.

3. Scandiani M, Luque A, Razori M, Ciancio Casalini L, Aoki T (2014) Metabolic profiles of soybean roots during early stages of Fusarium tucumaniae infection. J Exp Bot.

4. Hartley S, Eschen R, Horwood J, Gange AC, Hill EM (2014) Infection by a foliar endophyte elicits novel arabidopside-based plant defence reactions in its host, Cirsium arvense. New Phytol.

5. Wojakowska A, Muth D, Narożna D, Mądrzak C, Stobiecki M, et al. (2013) Changes of phenolic secondary metabolite profiles in the reaction of narrow leaf lupin (Lupinus angustifolius) plants to infections withColletotrichum lupini fungus or treatment with its toxin. Metabolomics 9: 575-589.

6. Hijaz FM, Manthey JA, Folimonova SY, Davis CL, Jones SE, et al. (2013) An HPLC-MS characterization of the changes in sweet orange leaf metabolite profile following infection by the bacterial pathogen Candidatus Liberibacter asiaticus. PLoS One.8: e79485.
7. Oliveira H, Barros A, Delgadillo I, Coimbra M, Santos C (2009) Effects of fungus inoculation and salt stress on physiology and biochemistry of in vitro grapevines: Emphasis on sugar composition changes by FT-IR analyses. Envir \& Exp Bot 65: 1-10.

8. Hong YS, Martinez A, Liger-Belair G, Jeandet P, Nuzillard JM, et al. (2012) Metabolomics reveals simultaneous influences of plant defence system and fungal growth in Botrytis cinerea-infected Vitis vinifera cv. Chardonnay berries J Exp Bot 63: 5773-85.

9. Ali K, Maltese F, Zyprian E, Rex M, Choi YH, et al. (2009) NMR metabolic fingerprinting based identification of grapevine metabolites associated with downy mildew resistance. J Agric Food Chem 57: 9599-606.

10. Lima M, Felgueiras ML, Graça G, Rodrigues JE, Barros A, et al. (2010) NMR metabolomics of esca disease-affected Vitis vinifera cv. Alvarinho leaves. J Exp Bot 61: 4033-42

11. Santos C, Fragoeiro S, Phillips A (2006) Physiological response of grapevine cultivars and a rootstock to infection with Phaeoacremonium and Phaeomoniella: An in vitro approach using plants and calluses. Sc Hort 107: 131-136.

12. Pereira SI, Figueiredo PI, Barros AS, Dias MC, Santos C, et al. (2014) Changes in the metabolome of lettuce leaves due to exposure to mancozeb pesticide. Food Chem 154: 291-8.

13. Rodriguez E, Azevedo R, Fernandes $P$, Santos $C$ (2011) $\mathrm{Cr}(\mathrm{VI})$ induces $D N A$ damage, cell cycle arrest and polyploidization: a flow cytometric and comet assay study in Pisum sativum. Chem Res Toxicol 24: 1040-7.

14. Silva S, Carnide O, Lopes P, Matos M, Pinto H, Santos C (2010) Differential aluminium changes on nutrient accumulation and root differentiation in an $\mathrm{A}$ sensitive vs. tolerant wheat. Env Exp Bot 68: 91-98. 